ECCOMAS

Proceedia
COMPDYN 2021

$8^{\text {th }}$ ECCOMAS Thematic Conference on Computational Methods in Structural Dynamics and Earthquake Engineering

M. Papadrakakis, M. Fragiadakis (eds.)

\title{
SEISMIC METAMATERIALS WITH TENSEGRITY ARCHITECTURE
}

\author{
Filipe Santos ${ }^{1 *}$, Narinder Singh ${ }^{2}$, Ada Amendola $^{2}$, Fernando Fraternali ${ }^{2}$ and Andrea \\ Micheletti $^{3}$ \\ ${ }^{1}$ Department of Civil Engineering \\ CERIS, NOVA School of Science and Technology-FCT NOVA, Universidade NOVA de Lisboa \\ 2829-516 Caparica, Portugal \\ e-mail: fpas@fct.unl.pt \\ 2 Department of Civil Engineering \\ University of Salerno, Via Giovanni Paolo II, 132 - 84084 Fisciano (Salerno), Italy \\ e-mail: $\{$ snarinder, adaamendola1, f.fraternali\}@unisa.it \\ ${ }^{3}$ Deptt. of Civil and computer Science Engineering \\ University of Rome, Tor Vergata, Italy \\ e-mail: micheletti@ing.uniroma2.it
}

\begin{abstract}
The class of nonlinear metamaterials with tensegrity architecture is particularly interesting, since the mechanical behavior of such systems can be effectively adjusted by acting on internal and external prestress, as well as the usual controls of geometry, topology, and mechanical properties of the members. This work is focused on the development of $3 D$ versions of the tensegrity braces (T-braces) recently proposed in the literature. The examined structures are aimed at forming novel seismic isolators, reinforcing seismic-resistant frames of steel and reinforced concrete buildings, as well as introducing the energy dissipation mechanisms of masonry walls, floor slabs, and junctions between seismic-resistant structures. This technology consists of lightweight and high-strength systems with re-centering capabilities formed by a variety of tensegrity systems. The tensegrity bracing systems incorporate superelastic shape memory bars/wires acting as pre-tensioned dissipative elements. The struts are prevented from buckling through the internal pretension of the braces via self-stress, and/or by contrasting them against the elements of the structure (external pre-tension). Using fractal geometry concepts, such systems can be designed through bio-inspired self-similar divisions of tensegrity brace modules to enhance both the energy dissipation capacity and the buckling resistance of the bracing system. Their development will significantly impact currently available techniques for the seismic protection of masonry and framed structures.
\end{abstract}

Keywords: metamaterials, Seismic isolators, Tensegrity, Shape memory alloys. 


\section{INTRODUCTION}

A novel area of research has emerged over the last few years regarding the highly nonlinear dynamics of mechanical metamaterials formed by assembling tensegrity units and lumped masses [4]. Experimental and theoretical studies have shown that the geometrically non-linear response of several tensegrity units may gradually change from stiffening to softening, depending on mechanical, geometrical, and prestress variables. Such behavior, which can be dynamically tuned, supports highly-controllable bandgap and solitary wave dynamics. For this particular reason, various tensegrity devices are being designed also for seismic protection [5]. A Tensegrity System is defined as a set of rigid bodies (subject to compression) that can be stabilized (therefore balanced) by a set of cables (or tense elements), in the absence of external forces [6]. The strings are in pretension and that pretension helps the system to be in equilibrium. There are several known examples of tensegrity structures, for example in nature (the bone-tendon system), in the art (for aesthetic beauty), in architecture (for their properties. These structures are nowadays usually referred to as "mechanical metamaterials". It has been found that linear elastic meta-materials can exhibit peculiar behaviors in the dynamical regime, like,e.g., negative effective elastic moduli, negative effective mass density, phononic band gaps, to name just a few examples [7]. The seismic response of strongly non-linear meta-materials has been particularly investigated in this article. The tensegrity structures can be 3D printed it is the system comprises of the bars and the strings. The strings are in tension and bars in compression. When we try to change the position, the strings experience some forces which put more compression on the bars and the strings face more tension These systems which are useful in dissipating the seismic energy are called passive energy dissipation devices. Various designs were studied before adopting the scissor-Jack design (SJD) by replacing the viscous damper with the SMA wires [6]. The reason behind SJD is it has magnification factors substantially greater than unity. Toggle-brace-damper $(\mathrm{C} \& \mathrm{~d})$ systems can also achieve the magnification factor greater than the unity. Viscous dampers used in these systems are pretty heavy [8] -[9]. To reduce the weight and to enhance the amplification, shape memory alloy (SMA) wires have been used in the current version of seismic devices. These SMA wires act as energy dissipators inside the braces because of their shape memory effect and super-elastic effect without having major deformation [11]. When stretched, the energy is dissipated in a considerable amount [12]- [14]. Further SMA effect of the wire amplifies the even infinitesimal movement in bars thus acting as sensors for energy dissipation device. In this article, two different types of seismic systems have been described one is named a 2D bracing system and another is 3D bracing system. In addition to the design, the experimental analysis of the 2D and 3D braces has been discussed in the next sections. It has been seen that 3D braces are far more efficient in terms of stability and dissipation because of the out-of-plane energy dissipation and the SMA effect of the strings [10].

\section{Materials and methods}

The key focus of this study was on the design and development of an anti-seismic system based on SMA wires. Furthermore, as discussed above, SMA wires have been used as restitution components instead of liquid viscous dampers to serve as passive energy dissipation (PED) equipment. The dissipation in this system is solely elastic due to the peculiar nature of the SMA wires. In this study, two types of bracing systems have been studied, namely the 2D bracing system (Figure. 1) and the 3D bracing system. In the 2D bracing method, the SJD model was used as shown in Figure 1 and was studied for its effectiveness. It was able to dissipate energy, 
but some limitations were observed. To overcome these drawbacks, particularly instability, less energy dissipation compared to the 3D brace model, and amplification, a 3D tensegrity bar model has been developed and theoretical results discussed in the following sections.

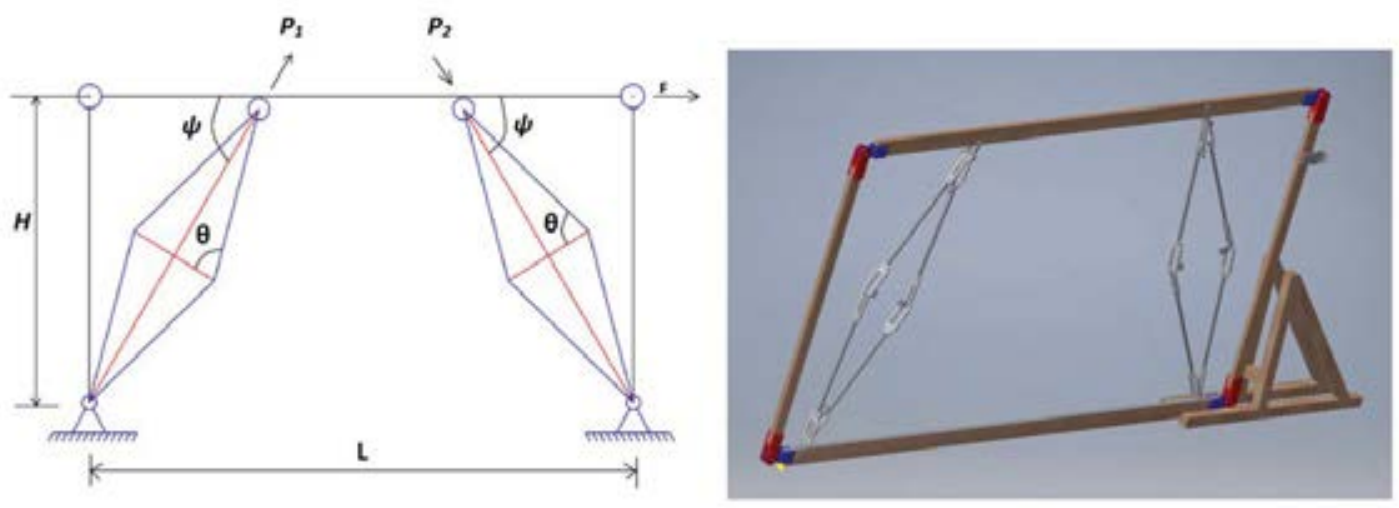

Figure 1: SMAD braces inserted into a frame structure

\section{Anti-seismic response of developed 2D bracing system}

After the design and development of the 2D tensegrity bar, theoretical tests were performed to access the necessary information. In Figure. 2 of Ref. [3] the amplification properties of the $2 \mathrm{D}$ tensegrity bar have shown and quite high amplification has been seen. In this system of braces, the system can achieve extreme position, unlike the liquid viscous dampers. Further, displacement in both the directions i.e, lateral and longitudinal directions has been seen, which makes it a better energy dissipation device by dissipation a fair amount of energy. In this, $u$ and $\mathrm{v}$ represent the longitudinal and the lateral displacements of the framework. In a special case of $u / v=\tan \theta, \beta$ approaches to 0 i.e, $\beta \rightarrow 0$, which signifies the negligible displacement from reference or original configuration. The variation of $u / v$ with $\theta$ for different values of $\beta$ is depicted in ref [3]. where $u$ and $v$, denote the longitudinal and the transverse displacements of the structure respectively.

Also, in Figure 3 of Ref. [3], the experimental and theoretical results were obtained and compared. It can be observed that the experimental and theoretical results are nearly identical. It is worth noting that minute residual strains are observed at unloading (not greater than 0.03 max) in experimental conditions.

\section{Anti-seismic response of developed 3D bracing system}

As discussed in previous sections, the extension to this work by designing a novel 3D tensegrity brace has been provided (see Figure. 2). Both physical and constitutive models have been developed and tested to characterize the force-displacement behavior of the bracing system. Due to the 3D shape of this configuration, it is possible to obtain a tensegrity element with increased stability towards out-of-plane buckling, and more effective in terms of energy dissipation. Researchers have found that triangular cross-section is by far the most effective configuration for a tensegrity element, where $\mathrm{N}=3$ [10].

Next, we show some numerical simulations of a 3D tensegrity bracing system, which enabled us to characterize its force-displacement response to cyclic loading (Fig. 3). In Figure. 4) it is displayed the geometric layout of the tested bracing. For further information about the 


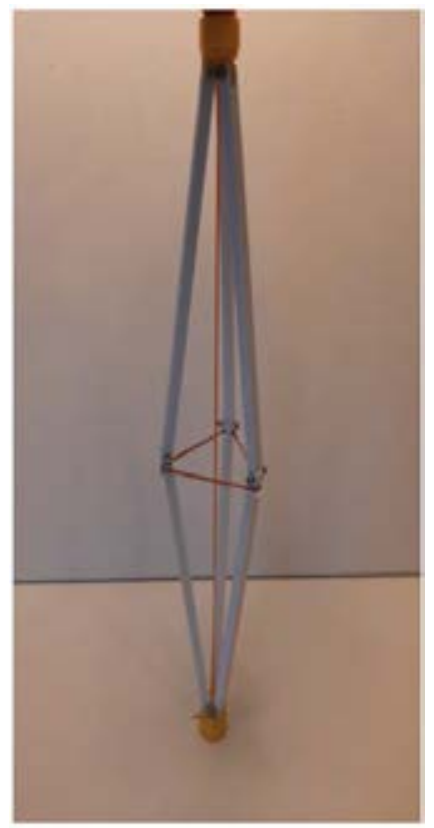

Figure 2: 3D model of the tensegrity bracing

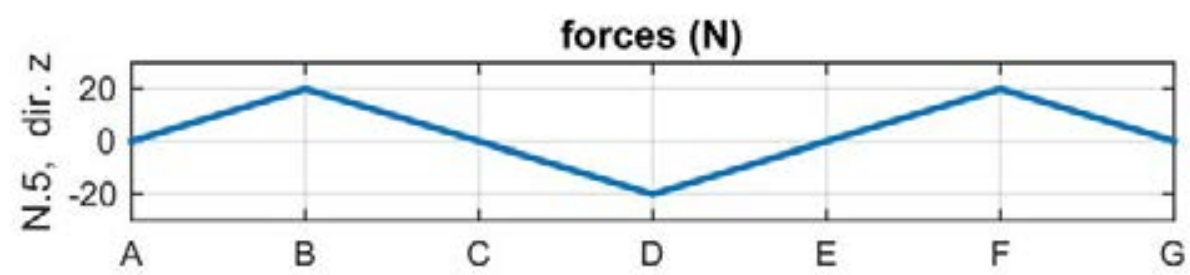

Figure 3: Mechanical loading cycle.

numerical implementation which was used for these simulations please refer to [15].

An initial pre-strain is introduced in the SMA wires (State A), prior to the implementation of the mechanical loading cycle, shown in Figure. 3. In Figure. 5 we show a compilation of the stress-strain-temperature responses of the SMA wires acting as ties in the 3D tensegrity bracing, during the imposed loading cycle. We assume isothermal conditions during the mechanical cycle, for an ambient temperature of $20^{\circ} \mathrm{C}$. Finally, in Figure. 6 we show the force-displacement plot for the proposed 3D tensegrity bracing, during the mechanical loading. Due to the fact that the horizontal (cables 1) and vertical (cable 2) ties are working in phase opposition, it is possible to obtain a wide superelastic hysteresis, highlighting the great energy dissipating capabilities of the proposed sytem.

\section{CONCLUSIONS}

In present work a novel method of developing seismic bracing systems with tensegrity architecture has been shown. Computational calculations of 2D and 3D tensegrity braces have been carried out to prove the effectiveness of the proposed systems. This research work is an extension of previous research focused on the development of a 2D PED device with tensegrity architecture incorporating SMA wires, which is a competitive alternative to viscous damper systems. SMA effects of the restitution elements has been proved to be very effective in terms 

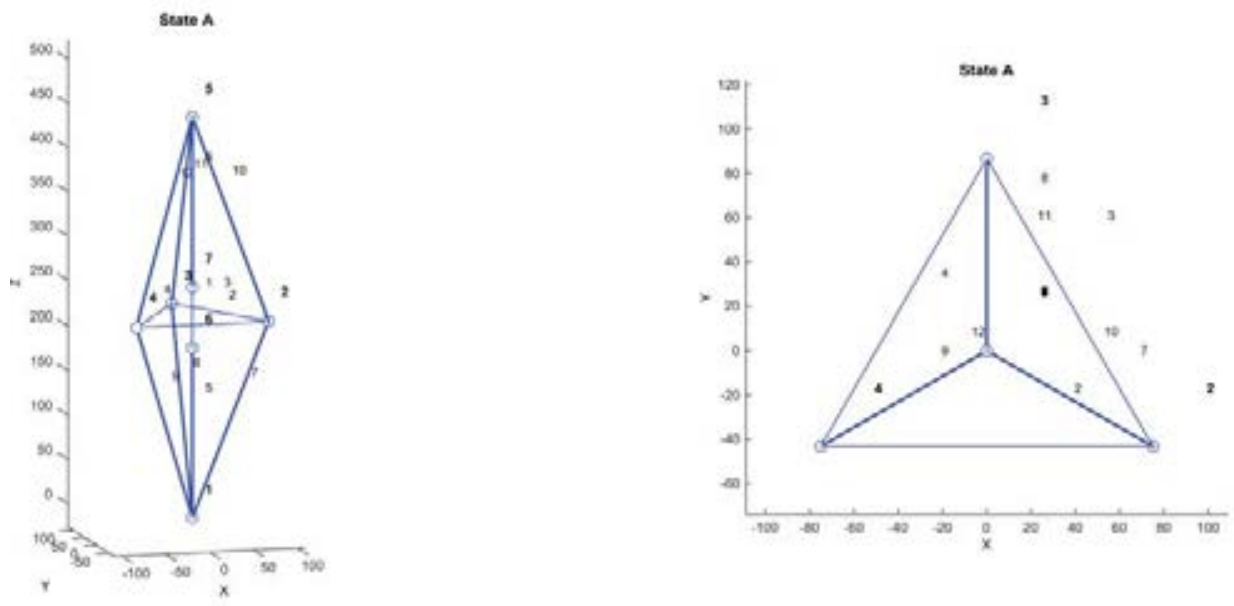

Figure 4: 3D numerical model of the tensegrity bracing (3D view and top view).

Cable 1

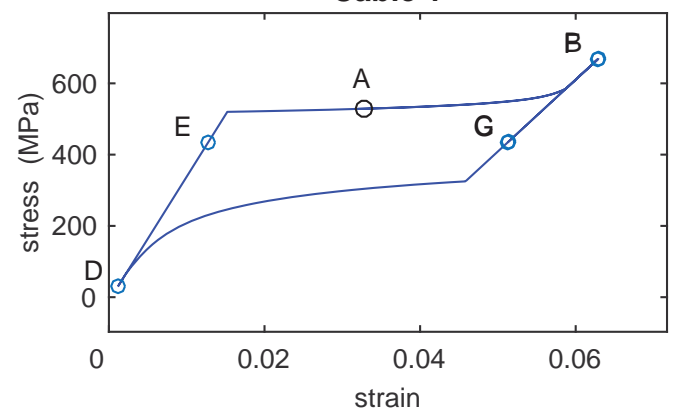

Cable 2

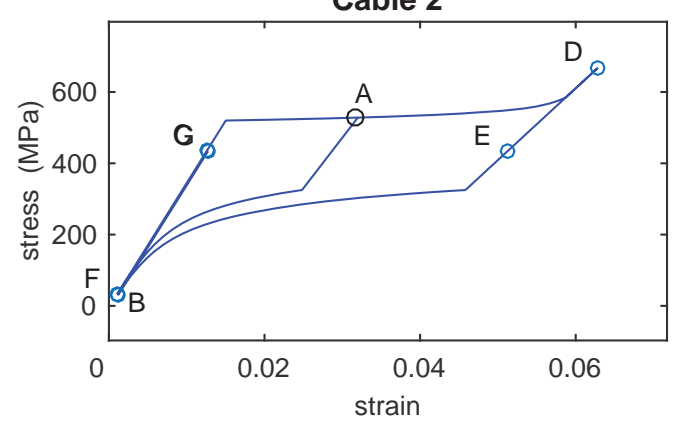

Cable 1

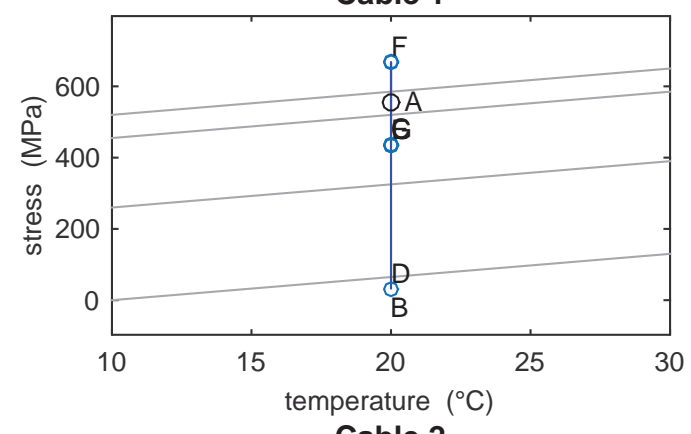

Cable 2

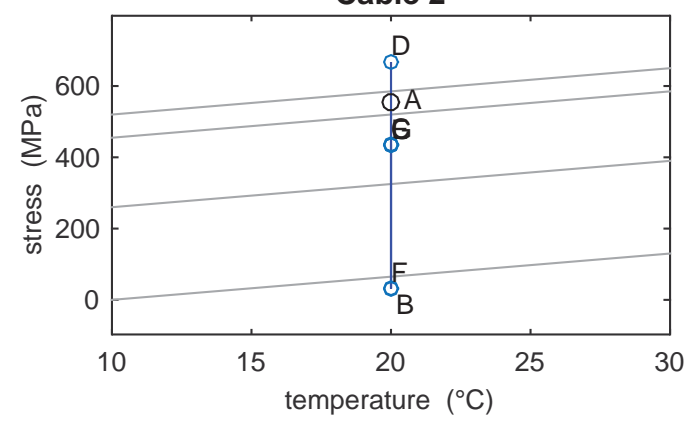

Figure 5: Stress-strain-temperature response of the SMA ties. 


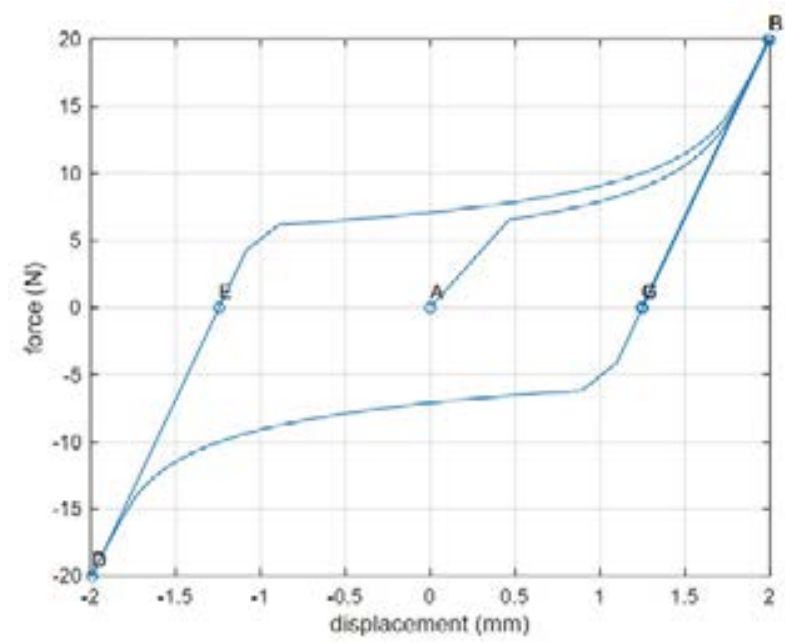

Figure 6: Force-displacement responses of a model frame reinforced with symmetrical SMAD braces.

of the displacement amplification factor and the energy dissipation capacity, because of the super-elastic properties of the SMA wires without experiencing permanent deformations. A 3D tensegrity bracing has been developed to multiply the existing effectiveness, such as energy dissipation and the stability of the bars. It has been seen from the theoretical results published in Ref. [3] that the amplification factor of the 2D brace is enhanced when the SMA wires are incorporated. In addition, in the case of the 3D brace, it is possible to obtain even more important energy dissipation features with additional lateral buckling restraints, as shown in the Figure. 6. The non viscous nature of the SMA wires makes the system lightweight and provide the opportunity to achieve extreme positions of the bar i.e., when the aspect angle is nearly 90 degrees. These features of the tensegrity bracing system would be very effective in technical applications, and indeed future steps are being taken to develop real scale models to be validated against experimental results by using various other meta materials and polymer materials and techniques [16]-[19].

\section{REFERENCES}

[1] A. Micheletti, G. Ruscica, F. Fraternali On the compact wave dynamics of tensegrity beams in multiple COMPDYN 2021 dimensions. Non linear dynamics, 98, 2737-2753, 2019.

[2] F. Santos, G. Benzoni, F. Fraternali, Seismic performance of superelastic tensegrity braces. Ingegneria sismica/international journal of earthquake engineering,36(3), 20-37, 2019.

[3] F. Fraternali, F. Santos, Mechanical modeling of superelastic tensegrity braces for earthquake-proof structures. Extreme mechanics letters, 33, 100578, 2019.

[4] F. Fraternali, G. Carpentieri, A. Amendola, R.E. Skelton, \& V. F. Nesterenko, V. F. Multiscale tunability of solitary wave dynamics in tensegrity metamaterials. Applied Physics Letters, 105(20), 201903, 2014.

[5] B. Basu, O. S. Bursi, F. Casciati, S. Casciati, A. E. Del Grosso, M. Domaneschi , ... \& J. Rodellar, A European Association for the Control of Structures joint perspective. 
Recent studies in civil structural control across Europe. Structural Control and Health Monitoring, 21(12), 1414-1436, 2014.

[6] AN. Sigaher, MC. Constantinou, Scissor-jack-damper energy dissipation system, Earthquake Spectra,19(1), 133-158, 2003.

[7] F. Fraternali, F. Santos, Mechanical modeling of superelastic tensegrity braces for earthquake-proof structures, Extreme Mechanics Letters, 33, 100578, 2019.

[8] M. Dolce, R. Marnetto, Passive seismic devices based on shape memory alloys, 12th World Conference on Earthquake Engineering, 1-8, 2000.

[9] Z. Xu, AK. Agrawal, WL. He, P. Tan, Performance of passive energy dissipation systems during near-field ground motion type pulses. Engineering Structure, 29(2), 224-236, 2007.

[10] RE. Skelton, MC. de Oliveira, Tensegrity Systems, Springer, 2010.

[11] V. Mercuri, Shape memory alloy strands: Conventional 3D FEM modeling and simplified models, MSc Thesis, University of Pavia, 2014.

[12] A. Alipour, M. Kadkhodaei, \& M. Safaei, Design, analysis, and manufacture of a tension-compression self-centering damper based on energy dissipation of pre-stretched superelastic shape memory alloy wires. Journal of Intelligent Material Systems and Structures, 28(15), 2129-2139, 2017

[13] R. DesRoches, \& M. Delemont, Seismic retrofit of simply supported bridges using shape memory alloys. Engineering Structures 24(3), 325-332, 2002.

[14] D. Liang, Y. Zheng, C. Fang, M. C. Yam, \& C. Zhang, Shape memory alloy (SMA)-cablecontrolled sliding bearings: development, testing, and system behavior. Smart Materials and Structures 29(8), 08500, 2002.

[15] A. Micheletti, F. Amarante dos Santos \& P. Sittner, Superelastic tensegrities: matrix formulation and antagonistic actuation. Smart Materials and Structures 27(10), 105028, 2018 .

[16] N. Singh, R. Singh, I.P.S. Ahuja, On Development of Functionally Graded Material Through Fused Deposition Modelling Assisted Investment Casting from Al2O3/SiC Reinforced Waste Low Density Polyethylene. Trans Indian Inst Met 71, 2479-2485, 2018.

[17] R. Miranda, E. Babilio, N. Singh, F. Santos, F. Fraternali, . Mechanics of smart origami sunscreens with energy harvesting ability. Mechanics Research Communications 105, $103503,2020$.

[18] R. Kumar, J. S. Chohan, R. Kumar, A. Yadav, N. Singh, Hybrid fused filament fabrication for manufacturing of Al microfilm reinforced PLA structures. Journal of the Brazilian Society of Mechanical Sciences and Engineering 42(9), 1-13, 2020.

[19] I. Farina, N. Singh , F. Colangelo, R. Luciano , G. Bonazzi F. Fraternali High-Performance Nylon-6 Sustainable Filaments for Additive Manufacturing. Materials 12(23), 3955, 2019. 